\title{
Transfer of hematopoietic stem cells encoding autoantigen prevents autoimmune diabetes
}

\author{
Raymond J. Steptoe, Janine M. Ritchie, and Leonard C. Harrison \\ Autoimmunity and Transplantation Division, The Walter and Eliza Hall Institute of Medical Research, \\ Royal Melbourne Hospital, Parkville, Victoria, Australia
}

\begin{abstract}
Bone marrow or hematopoietic stem cell transplantation is a potential treatment for autoimmune disease. The clinical application of this approach is, however, limited by the risks associated with allogeneic transplantation. In contrast, syngeneic transplantation would be safe and have wide clinical application. Because $\mathrm{T}$ cell tolerance can be induced by presenting antigen on resting antigen-presenting cells (APCs), we reasoned that hematopoietic stem cells engineered to express autoantigen in resting APCs could be used to prevent autoimmune disease. Proinsulin is a major autoantigen associated with pancreatic $\beta$ cell destruction in humans with type 1 diabetes (T1D) and in autoimmune NOD mice. Here, we demonstrate that syngeneic transplantation of hematopoietic stem cells encoding proinsulin transgenically targeted to APCs totally prevents the development of spontaneous autoimmune diabetes in NOD mice. This antigen-specific immunotherapeutic strategy could be applied to prevent T1D and other autoimmune diseases in humans.
\end{abstract}

J. Clin. Invest. 111:1357-1363 (2003). doi:10.1172/JCI200315995.

\section{Introduction}

Bone marrow (BM) or hematopoietic stem cell (HSC) transplantation has recently been used to treat clinically severe autoimmune disease (1). In preclinical animal models, effective treatment of spontaneous autoimmune disease requires transplantation of BM or HSCs from disease-resistant strains. To date, this has been achieved by allogeneic BM transplantation (BMT) (2-6) leading to full or mixed chimerism $(7,8)$. However, the requirement for cytotoxic conditioning of the host and the risk of graft rejection (9) or graft-versushost disease (10) render this approach unsuitable for widespread clinical application. Transplantation of syngeneic HSCs would avoid these problems and represent a novel, safe, and effective strategy for preventing autoimmune disease.

Antigen presentation by resting antigen-presenting cells (APC), either B cells $(11,12)$ or dendritic cells $(13$, 14 ), is a means of inducing T cell tolerance. Since APCs are derived from HSCs we reasoned that HSCs encoding autoantigen targeted to APCs could be used to

Received for publication May 24, 2002, and accepted in revised form February 26, 2003.

Address correspondence to: Leonard C. Harrison, The Walter and Eliza Hall Institute of Medical Research, Post Office, Royal Melbourne Hospital, Parkville, 3050 Victoria, Australia. Phone: 61-3-9345-2460; Fax: 61-3-9347-0852;

E-mail: harrison@wehi.edu.au.

Conflict of interest: The authors have declared that no conflict of interest exists.

Nonstandard abbreviations used: antigen-presenting cell (APC); type 1 diabetes (T1D); bone marrow (BM); hematopoietic stem cell (HSC); BM transplantation (BMT); phycoerythrin (PE); peripheral blood leukocyte (PBL); hematopoietic progenitor cell (HPC); ovalbumin (OVA); stimulation index (SI); glutamic acid decarboxylase (GAD); dendritic cell (DC). induce tolerance and thereby prevent autoimmune disease. Type 1 diabetes (T1D) is an autoimmune disease in which insulin-secreting $\beta$ cells in the pancreatic islets are destroyed by autoreactive $\mathrm{T}$ cells. In humans with T1D and in the NOD mouse model of $\mathrm{T} 1 \mathrm{D}$, several lines of evidence indicate that proinsulin is a key autoantigen that drives $\beta$ cell destruction (15-18). To test whether syngeneic HSCs encoding autoantigen could prevent autoimmune disease, we transplanted into wild-type NOD mice BM or HSCs from NOD mice in which proinsulin was transgenically targeted to APCs.

\section{Methods}

Mice. NOD, NOD-PI, NOD.scid, and congenic NOD CD45.2 (Ly5.2) mice were bred under specific pathogen-free conditions in the Walter and Eliza Hall Institute central breeding facility. NOD-PI mice, transgenic for mouse proinsulin II under the control of an MHC class II (I- $\left.E_{\alpha}{ }^{k}\right)$ promoter (18), were used after breeding to homozygosity. Because NOD females have the highest incidence of spontaneous autoimmune diabetes, they were used as recipients and BM donors.

Antibodies and flow cytometry. Flow cytometric analysis was performed as previously described (19). The following $\mathrm{mAb}$ 's were purified from tissue culture supernatants and conjugated in house: anti-CD3 (KT3), CD11b (5C6 or M1/70), B220 (RA3.6B2), Gr-1 (RB68C5), Ly-76 (TER-119), and c-kit (ACK-2). Anti-CD3 (145-2C11), SCA-1 (E13-161.7), CD45.1 (A20), and CD45.2 (104) were from Pharmingen (San Diego, California, USA). mAb's to CD4 (CT-CD4), CD8 $\alpha$ (CTCD8a), and FITC, phycoerythrin (PE), and Tricolor streptavidin conjugates were from Caltag Laboratories (Burlingame, California, USA). For analysis of peripheral blood leukocytes (PBLs), mice were bled by retro- 
orbital venous sinus puncture with a fine glass capillary tube. Blood was collected in Alsever's anticoagulant, erythrocytes were lysed, and leukocytes were stained and analyzed by flow cytometry. Leukocyte number determined with a hemocytometer was calibrated according to the blood volume obtained. Spleens were pressed through stainless steel mesh, and cells were suspended in RPMI containing $10 \%$ FCS. Staining was performed as described previously (19). Briefly, cells were "preblocked" by incubation in "blocking mix" $(10 \% \mathrm{vol} / \mathrm{vol}$ normal mouse serum and $10 \% \mathrm{v} / \mathrm{v}$ anti-CD16/32 [2.4G2] tissue culture supernatant) at $4^{\circ} \mathrm{C}$ for 5 minutes. Cells were incubated with FITC-, PE-, or biotinconjugated primary antibodies at $4^{\circ} \mathrm{C}$ for 30 minutes in blocking mix, washed in PBS/1.5\%FCS, and incubated with streptavidin-FITC or streptavidin-PE (Caltag Laboratories) as required before a final wash. For cytometry, samples were analyzed on a FACScan (Becton Dickinson, Mountain View, California, USA), and gates were set to include live cells on the basis of forward and side scatter profiles. For analysis, $10^{4}$ forward/side scatter gated cells were collected.

BM preparation and transfer. Mice (8-12 weeks old) were euthanized, and femurs and tibias were collected into cold PBS. BM was flushed with ice-cold PBS containing $2.5 \%$ FCS (F2.5) (Trace Scientific, Melbourne, Australia), and erythrocytes were removed by lysis in $\mathrm{NH}_{4} \mathrm{Cl} /$ Tris buffer. BM was washed in F2.5 and collected by centrifugation. For $\mathrm{T}$ cell depletion, $\mathrm{BM}$ was resuspended in $\mathrm{F} 2.5$, incubated with anti-CD3 $\mathrm{mAb}(\mathrm{KT} 3,5 \mu \mathrm{g} / \mathrm{ml})$ for 30 minutes at $4^{\circ} \mathrm{C}$, and then washed in F2.5. Antibodylabeled cells were depleted with anti-rat IgG immunomagnetic beads (Dynabeads, Dynal Biotech, Melbourne, Australia). For sorted HSCs or hematopoietic progenitor cells (HPCs), lineage marker-positive cells were depleted by immunomagnetic beads with a mix of FITCconjugated lineage-specific mAb (KT3, M1/70, RA3.6B2, RB6-8C5, TER-119) at predetermined optimal concentrations. Remaining cells were labeled with anti-c-kitPE. For HSC isolation, lineage-depleted cells were also costained with anti-SCA-1-biotin, washed, and stained with streptavidin-Tricolor. $\mathrm{Lin}^{-} / \mathrm{c}-\mathrm{kit}^{+} / \mathrm{SCA}-1^{+}$(HSC) or $\mathrm{lin}^{-} / \mathrm{c}-\mathrm{kit}^{+}$(HPC) cells were collected by sterile sorting (FACSII, Becton Dickinson). Female NOD mice, as recipients of whole $\mathrm{BM}$ were irradiated at 4 weeks of age with a single dose of $800 \mathrm{cGy}$ (Theratron ${ }^{60} \mathrm{Co}$, Theratronics, Kanata, Ontario, Canada) and as recipients of other cells with a total of 950 cGy in two equal doses 2-3 hours apart. Cells $\left(10^{7} \mathrm{BM}\right.$ or T cell-depleted BM cells, unless stated otherwise) were suspended in PBS and injected intraperitoneally in $250 \mu \mathrm{l}$ or intravenously in $100 \mu \mathrm{l}$ for HSCs $\left(10^{3}\right)$ and HPCs $\left(2.5 \times 10^{4}\right) 1-3$ hours after irradiation. Irradiated mice were maintained on neomycin-supplemented drinking water for 3 weeks after BMT. Any mice showing signs of physical distress in the immediate post-BMT period were euthanized and excluded from analysis.

Monitoring for diabetes. The urine of the mice was tested weekly for glucose with Diastix test strips (Bayer,
Pymble, New South Wales, Australia). In glycosuric mice, blood glucose was measured with a meter (AccuChek, Roche Diagnostics, Castle Hill, New South Wales, Australia). Mice were considered diabetic when two consecutive blood glucose readings were greater than $12.0 \mathrm{mM}$. Mice were euthanized when diabetic or showing sign of physical distress.

Assessment of insulitis and sialitis. Pancreata and submandibular glands were removed from euthanized mice, placed in Bouin's fixative for 24 hours, and then transferred to $70 \%$ ethanol. Fixed tissues were embedded in paraffin, and hematoxylin- and eosin-stained sections separated by $250-300 \mu \mathrm{m}$ were prepared. Cellular infiltration of pancreatic islets (insulitis) was scored in a masked fashion as described (20), and the number of inflammatory foci in submandibular glands was counted and expressed as a mean per section.

Assessment of NOD-PI cells in recipient lymphoid tissue. Spleens were collected from treated mice at the onset of clinical diabetes or the termination of the experiment (250 days of age). Genomic DNA was prepared from spleens by proteinase $\mathrm{K}$ digestion and phenol/chloroform extraction. Proinsulin II transgene DNA was quantified by real-time PCR using a LightCycler (Roche Diagnostics, Mannheim, Germany). Real-time PCR was performed on $25 \mathrm{ng}$ of genomic DNA with target-specific (I- $\mathrm{E}_{\alpha}{ }^{\mathrm{k}}$ promoter-proinsulin II) primers (forward, ttgttaattctgcctcagtctgcg; reverse, tgtcccaagtgtgaagaaaaccag) and standard-specific ( $\beta$-actin) primers (forward, tgtatgcctctggtcgtacc; reverse, caacgtcacacttcatgatgg). Amplicons were detected by SYBR green 1 dye binding (Molecular Probes, Eugene, Oregon, USA). Normalized ratios of target/standard DNA were obtained with Relative Quantitation software (Roche Diagnostics) using PCR efficiency curves generated from DNA dilution series. Genomic DNA prepared from a single NOD-PI mouse was used as the internal standard (calibrator) in each real-time PCR assay. The proportion of NOD-PIderived cells was determined as the ratio of target (NOD-PI transgene) DNA to standard ( $\beta$-actin) DNA normalized to the NOD-PI calibrator. The detection limit of the PCR assay was less than 5 pg of target DNA, equivalent to $0.025 \%$ NOD-PI-derived cells.

BM engraftment was also quantified by transferring NOD CD45.2 BM (107 cells) to irradiated (800 cGy) wild-type NOD (CD45.1) mice, as described above. The proportion of donor CD45.2 cells was analyzed in PBLs and spleen cells by flow cytometry.

$T$ cell recall response. Mice were immunized subcutaneously in the flank with $100 \mu \mathrm{g}$ of ovalbumin (OVA) (Grade V, Sigma-Aldrich, St Louis, Missouri, USA) in complete Freund's adjuvant (Difco, Detroit, Michigan, USA). Spleens collected 14 days later from euthanized mice were pressed through stainless steel mesh, and cells were suspended in RPMI (GIBCO, Rockville, Maryland) containing 10\% FCS (Trace Scientific), $10^{-3}$ $\mathrm{M}$ sodium pyruvate, $10^{-4} \mathrm{M}$ nonessential amino acids (GIBCO), $2 \times 10^{-3} \mathrm{M}$ glutamine, and $5 \times 10^{-5} \mathrm{M} 2$-mercaptoethanol (Sigma-Aldrich). Splenocytes were plated 
in triplicate $\left(2.5 \times 10^{5}\right.$ cells per well, $200 \mu \mathrm{l}$, 96-well flatbottom plates) in the absence or presence of OVA (100 $\mu \mathrm{g} / \mathrm{ml})$. Cells were harvested on day 4 onto glass filter mats. ${ }^{3} \mathrm{H}$-thymidine $(1 \mu \mathrm{Ci}$ per well) was added during the final 18 hours of culture. Incorporated radioactivity reflecting cell proliferation was measured in a scintillation counter (Topcount, Packard, Groningen, The Netherlands), and results were expressed as mean stimulation index $(\mathrm{SI}) \pm \mathrm{SD}$.

Assays for immunity to islet antigens. Cellular immune responses were measured as ${ }^{3} \mathrm{H}$-thymidine uptake in spleen cell suspensions not exposed to red cell lysis buffer, as described above for the $\mathrm{T}$ cell recall response. Antigens were (pro)insulin $\mathrm{T}$ cell epitope peptides aa9-23 (21) and aa24-36 (22) (purchased from Auspep, Melbourne, Australia) at $20 \mu \mathrm{g} / \mathrm{ml}$ and recombinant human glutamic acid decarboxylase (GAD) 65 (produced in house) at $20 \mu \mathrm{g} / \mathrm{ml}$; anti-CD3 monoclonal antibody $145-2 \mathrm{C} 11$ at $2.5 \mu \mathrm{g} / \mathrm{ml}$ was used as a positive control. Insulin antibodies in $5 \mu \mathrm{l}$ of serum were measured at a 1:5 dilution (assay volume, $50 \mu \mathrm{l}$ ) by binding to ${ }^{125} \mathrm{I}$-insulin $(20,000 \mathrm{cpm})$ in the presence and absence of $300 \mu \mathrm{g} / \mathrm{ml}$ unlabeled human insulin for 3 days at $4^{\circ} \mathrm{C}$, followed by precipitation of complexes with protein A-sepharose, washing, and counting in a $\gamma$ counter. Results were calibrated against a reference serum dilution curve, and the cutoff for positivity was $3 \mathrm{pM}$. The assay has been described and evaluated in an international workshop (23).

Statistical analysis. Comparison of Kaplan-Meier survival curves was performed using the log-rank test (GraphPad Prism, GraphPad Software Inc., San Diego, California, USA). Insulitis scores between BMT groups were compared by Student's $t$ test.

\section{Results}

Transplantation of NOD-PI bone marrow prevents diabetes. Whole BM was transplanted from NOD or NOD-PI mice to 4-week-old irradiated female NOD recipients. In recipients of NOD BM, the onset of diabetes was delayed slightly, but the overall incidence of diabetes $(7 / 12)$ was similar to that in untreated controls $(15 / 23)$ (Figure 1a). In contrast, in recipients of NOD-PI BM, diabetes was almost completely prevented $(1 / 16$ versus $7 / 12, P=0.003$ ) (Figure 1a). NOD mice have an inherently high risk of thymoma development that is exacerbated by impaired immune surveillance or exposure to ionizing radiation $(24,25)$. Exclusion of mice found to have thymomas at necropsy increased the proportion of mice with diabetes in both groups (NOD, 7/10; NOD-PI, 1/5), but the difference in the incidence of diabetes remained significant between groups $(P=0.041)$. The incidence of thymoma and the mean age at detection were not different between recipients of NOD $(16 / 57,202 \pm 28$ days $)$ or NOD-PI BM $(14 / 50,196 \pm 41$ days) for all experiments presented.

$T$ cell depletion does not modify the protective effect of transplanted NOD-PI bone marrow. In separate studies (R. J. Steptoe et al., unpublished observations), we found that mature $\mathrm{T}$ cells in NOD BM but not NOD-PI BM were capable of transferring diabetes to immune-deficient NOD.scid mice. Therefore, we tested whether development of diabetes after transfer of whole NOD or NOD-PI BM reflected the diabetogenic potential of transferred mature T cells. Whole BM $\left(40 \times 10^{6}\right.$ cells injected intraperitoneally) from NOD mice transferred diabetes to at least $50 \%$ of nonirradiated T cell-deficient NOD.scid mice, whereas no mice that received BM from NOD-PI mice developed diabetes. T cells in the BM may have either diabetogenic or regulatory function that could account for the differential ability of NOD and NOD-PI BM T cells to transfer disease to NOD.scid recipients. Therefore, to rule out any contribution of mature $\mathrm{T}$ cells transferred in BM, we tested the effect of transplanting T cell-depleted BM to irradiated 4-week-old mice. Recipients of T cell-depleted NOD BM developed diabetes at the same rate and with the same incidence as untreated controls (10/15 versus $7 / 12$ ) (Figure $1 b)$. In contrast, diabetes developed at a significantly lower frequency in recipients of T cell-depleted NOD-PI BM than in recipients of NOD BM (3/17 versus $10 / 15$, $P=0.003$ ) (Figure $1 \mathrm{~b}$ ). When mice with thymomas at necropsy were removed from the analysis, the
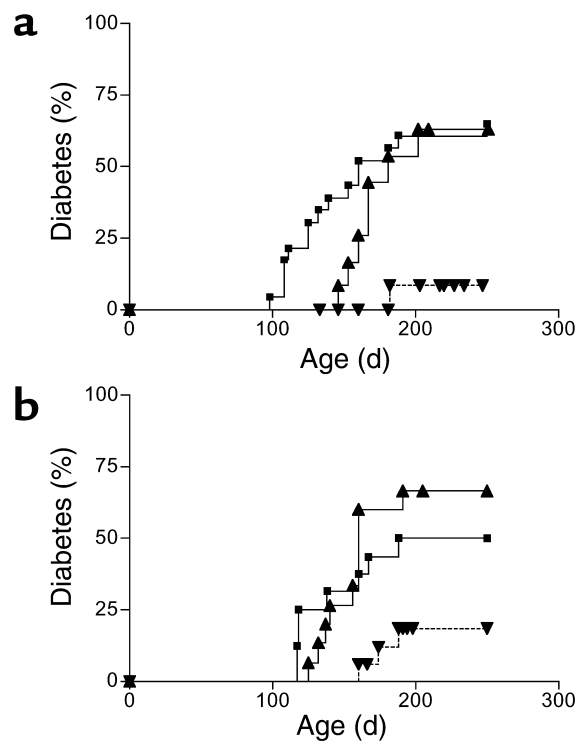

\section{Figure 1}

Transplantation of NOD-PI BM inhibits development of diabetes. (a) The incidence of diabetes was significantly reduced in recipients of NOD-PI BM (inverted triangles) as compared with NOD BM (triangles) $(P=0.003)$ or untreated NOD mice (squares) $(P=0.001)$. NOD $B M$ recipients did not differ from the untreated controls. Data are pooled from two experiments in which BMT from NOD-PI mice or NOD mice was performed in parallel. (b) The incidence of diabetes was significantly reduced in recipients of T cell-depleted NOD-PI BM (inverted triangles) as compared with T cell-depleted NOD BM (triangles) $(P=0.003)$ or untreated NOD mice (squares) $(P=0.036)$. NOD BM recipients did not differ from untreated controls. Data are pooled from three experiments in which BMT from NOD-PI or NOD mice was performed in parallel. Cumulative incidence of diabetes is shown on the $y$-axis and the age of mice in days on the $x$-axis. 

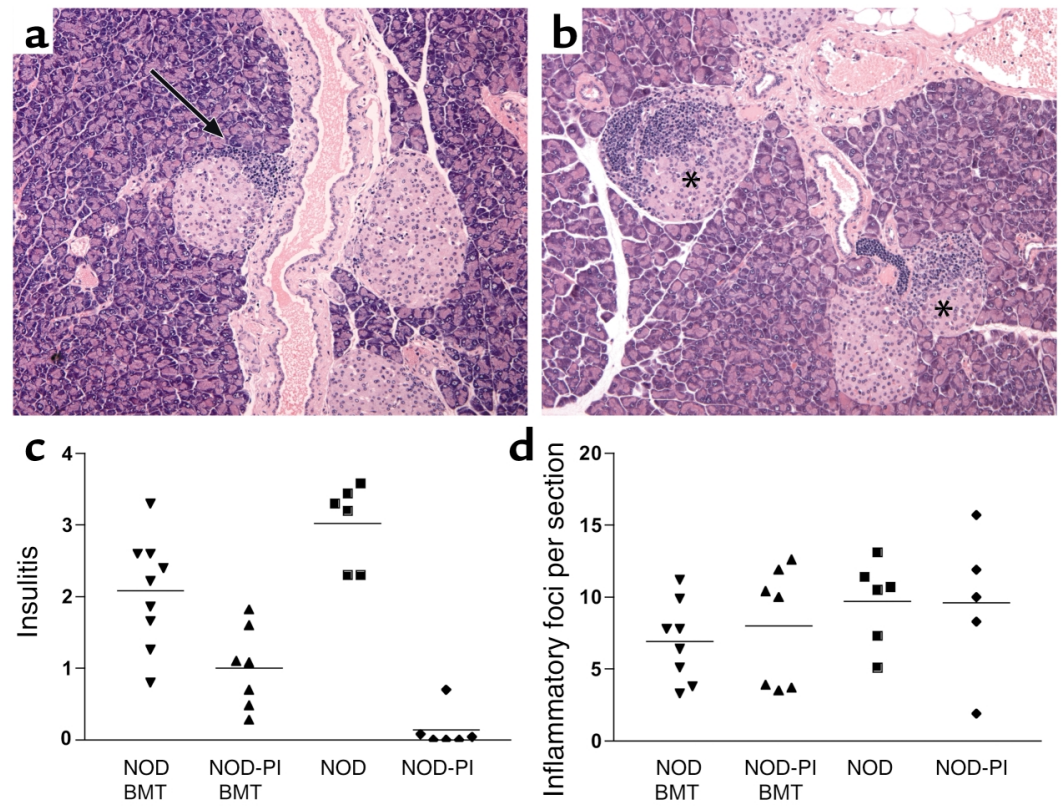

\section{Figure 2}

Transplantation of T cell-depleted NOD-PI BM prevents insulitis but not sialitis. (a) Islets free of inflammatory infiltrate (insulitis) were common in recipients of NOD-PI BM, and infiltration was restricted to the periphery of islets (arrow). (b) Extensively infiltrated islets (indicated by asterisks) were common in recipients of NOD BM. (c) The insulitis score was significantly reduced $(P=0.008)$ in recipients of $\mathrm{T}$ cell-depleted NOD-PI BM cells (triangles, $1.0 \pm 0.6$ ) as compared with NOD BM cells (inverted triangles, $2.1 \pm 0.8$ [means $\pm \mathrm{SD}$ ]). Data for age-matched NOD mice (squares) and NOD-PI mice (diamonds) are included for comparison. (d) The number of submandibular gland inflammatory foci (sialitis score) did not differ between BMT and untreated mice. Individual mouse scores are pooled from two experiments in which BMT from NOD and NOD-PI mice was performed in parallel (horizontal bar indicates mean). difference between groups remained statistically significant $(2 / 11$ versus $10 / 15, P=0.012)$.

Cellular immune infiltration of pancreatic islets (insulitis) was assessed 10 weeks after transplantation of T cell-depleted BM $\left(10 \times 10^{6}\right.$ cells $)$, when mice were approximately 100 days of age. In recipients of $\mathrm{T}$ cell-depleted NOD-PI BM, $54 \%$ of islets were free of insulitis, and mononuclear cell infiltration was restricted to the islet periphery (peri-insulitis) (Figure 2a). In contrast, in recipients of T cell-depleted NOD $\mathrm{BM}$, only $28 \%$ of islets were free of insulitis, and there was extensive infiltration into the islets (Figure 2b). These observations were reflected by a significantly reduced mean insulitis score in recipients of NOD-PI as compared with NOD BM ( $1.0 \pm 0.6$ versus $2.1 \pm 0.8$, $P=0.008$ ) (Figure 2c). In contrast to insulitis, mononuclear cell infiltration of the submandibular gland (sialitis) was similar in recipients of $\mathrm{T}$ celldepleted NOD-PI or NOD BM and age-matched unmanipulated controls (Figure 2d). This indicates that transfer of NOD-PI BM protects specifically against islet autoimmunity. Similar results were

\section{Figure 3}

Transfer of NOD-PI HSCs or HPCs prevents development of diabetes. (a) Diabetes was prevented in recipients of NOD-PI HSCs (inverted triangles) compared with recipients of NOD HSCs (triangles) $(P=0.019)$ or untreated NOD mice (squares) $(P=0.029)$. NOD HSC recipients and untreated NOD mice did not differ significantly. (b) The incidence of diabetes was significantly reduced in recipients of NOD-PI HPCs (inverted triangles) compared with recipients of NOD HPCs (triangles) $(P=0.035)$ or untreated NOD mice (squares) $(P=0.021)$. NOD HPC recipients and untreated NOD mice did not differ significantly. Data are from one (a) or two (b) experiments in which BMT from NOD-PI and NOD mice was performed in parallel. observed after transplanting half the amount of $\mathrm{T}$ cell-depleted BM $\left(5 \times 10^{6}\right.$ cells $)$.

Proinsulin-encoding HSCs and HPCs transfer diabetes prevention. HSCs $\left(\mathrm{lin}^{-} / \mathrm{c}-\mathrm{kit}^{+} / \mathrm{SCA}-1^{+}\right)$or HPCs $\left(\mathrm{lin}^{-} / \mathrm{c}-\mathrm{kit}^{+}\right)$ were sterile purified from NOD and NOD-PI BM, and small numbers of either were transplanted into irradiated 4-week-old recipients. Hematopoietic reconstitution was rapid, and PBL populations were restored by 8 weeks after BMT (data not shown). Diabetes was totally prevented in recipients of NOD-PI HSCs $(0 / 10)$ as compared with NOD HSCs (5/9) or untreated controls $(8 / 16)$ (Figure 3a). The incidence of diabetes was significantly reduced in recipients of NOD-PI HPCs $(2 / 14, P=0.035)$ as compared with NOD HPCs $(9 / 19)$ or untreated (8/16) controls (Figure $3 b)$.

Diabetes is inhibited in the presence of low levels of NOD-PI $B M$ engraftment. To determine the level of NOD-PI BM
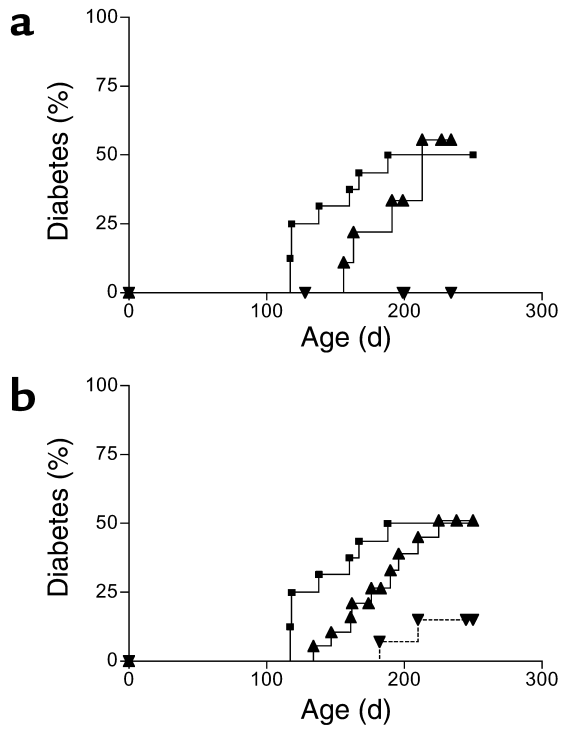
Table 1

Engraftment of NOD-PI-derived cells in recipient spleen

\begin{tabular}{|c|c|c|c|}
\hline & $\begin{array}{l}\text { NOD-PI cells } \\
(\%)^{A}\end{array}$ & $n$ & $\begin{array}{l}\text { Inhibition of diabetes } \\
\text { development }(\%)^{\mathrm{B}}\end{array}$ \\
\hline Whole BM & $4.6 \pm 4.2$ & 10 & 89 \\
\hline T cell-depleted BM & $5.1 \pm 5.4$ & 9 & 80 \\
\hline HPCs $\left(\operatorname{lin}^{-}, \mathrm{c}^{-} \mathrm{kit}^{+}\right)$ & $37.4 \pm 27.0$ & 11 & 73 \\
\hline HSCs $\left(\mathrm{lin}^{-}, \mathrm{c}-\mathrm{kit}^{+}, \mathrm{sca}-1^{+}\right)$ & $73.1 \pm 26.1$ & 7 & 100 \\
\hline
\end{tabular}

${ }^{A}$ Determined as the ratio of target (NOD-PI transgene) DNA to standard ( $\beta$-actin) DNA, which in mice receiving wild-type NOD BMT was less than $10^{-5}$. ${ }^{B}$ Calculated from data shown in Figures 1 and 3.

engraftment required to inhibit diabetes, real-time PCR was used to quantify NOD-PI-derived cells in spleens from mice transplanted with BM, HPCs, or HSCs. After intravenous transfer of $10^{3}$ purified HSCs, a substantial proportion (range, 33-100\%) of NOD-PI-derived cells was present in recipient spleens (Table 1), whereas after intraperitoneal transfer of whole or T cell-depleted BM, the proportion (range, $0.1-11.9 \%$ ) was lower (Table 1). These findings were validated by using congenic NOD CD45.2 mice as whole BM donors. The level of donor (CD45.2) engraftment in PBL $(9.0 \% \pm 5.6 \%, n=4)$ or spleen $(8.4 \% \pm 5.5 \%, n=6) 12$ weeks after intraperitoneal transfer of $\mathrm{T}$ cell-depleted $\mathrm{BM}$ was similar to that determined by real-time PCR. Although diabetes prevention was $100 \%$ at high levels of engraftment, a substantial reduction in diabetes incidence was still observed at low levels of engraftment (Table 1).

Diabetes prevention by NOD-PI bone marrow is not due to impaired immune reconstitution. To exclude the possibility that the protective effect of NOD-PI BMT was the result of impaired immune reconstitution, we first analyzed PBL populations. Ten to fourteen days after BMT, circulating leukocytes remained substantially reduced (Figure 4a). The proportions of $\mathrm{CD}^{+}$and $\mathrm{CD}^{+} \mathrm{T} \mathrm{lym}$ phocytes and $\mathrm{B} 220^{+} \mathrm{B}$ lymphocytes were reduced $50-75 \%, 25 \%$, and $80-85 \%$, respectively, relative to agematched controls, whereas the proportion of $\mathrm{CD} 11 \mathrm{~b}^{+}$ myeloid cells was increased approximately 2.5 -fold (data not shown). After 8 and 16 weeks, total PBLs (Figure 4a) and the relative proportion of PBL subsets (Table 2) were normal, indicating similar reconstitution between groups.

We then compared the ability of BMT recipients to mount a $\mathrm{T}$ cell-mediated immune response. Normal age-matched NOD mice and recipients of NOD or NOD-PI T cell-depleted BM were immunized with OVA 10 weeks after transplantation, when mice were approximately 100 days of age. Two weeks later, in vitro recall responses to OVA were identical in untreated mice and either transplant group (Figure 5). Transplantation of T cell-depleted NOD-PI BM was therefore not associated with evidence of impaired immune reconstitution. We could not, however, detect significant $\mathrm{T}$ cell-proliferative responses to (pro)insulin $\mathrm{T}$ cell epitope peptides aa9-23 or aa24-36 or to human GAD 65 in wild-type NOD mice or in NOD mice 12 weeks after NOD or NOD-PI BMT. Furthermore, the frequency of insulin autoantibodies in recipients of NOD (4/15) and NOD-PI (4/14) BM was no different (data not shown).

\section{Discussion}

The ability to transfer diabetes in the NOD mouse with $\mathrm{BM}$ or $\operatorname{HPC}(3,26,27)$ demonstrates not only the hematopoietic basis of disease but the potential to prevent autoimmune diabetes by manipulating hematopoiesis-derived cells. Indeed, replacement of the NOD hematopoietic compartment with that from diabetes-resistant strains by BMT prevents development of diabetes $(3,7)$. To date, however, effective BMT therapy has required generation of full- or mixed-allogeneic chimerism $(3,7,8,28)$ and is limited by toxic conditioning regimens and the risk of graft rejection and/or graft-versus-host disease. Syngeneic transplantation of gene-modified HSCs as described here is a novel approach to antigen-specific immunotherapy that advances the principle of regulating autoimmune disease from within the hematopoietic compartment.

Proinsulin, the prohormonal precursor of insulin, contains $\mathrm{T}$ cell epitopes implicated in human (29-31) and mouse $(21,32)$ T1D. By using NOD mice that express proinsulin transgenically targeted to APCs by an MHC class II promoter (18) as donors, we have been able to show that syngeneic BMT adoptively transfers protection against the development of autoimmune diabetes. Although recipients of BM from NOD-PI mice were substantially protected from diabetes, insulitis was occasionally observed and diabetes developed in a minority. This may be due to the persistence of a small number of radio-resistant pathogenic $T$ cells or to the emergence of $\mathrm{T}$ cells after BMT directed at islet antigens other than proinsulin. Complete protection after transfer of highly purified HSCs shows that diabetes prevention depends on the engraftment of multipotent

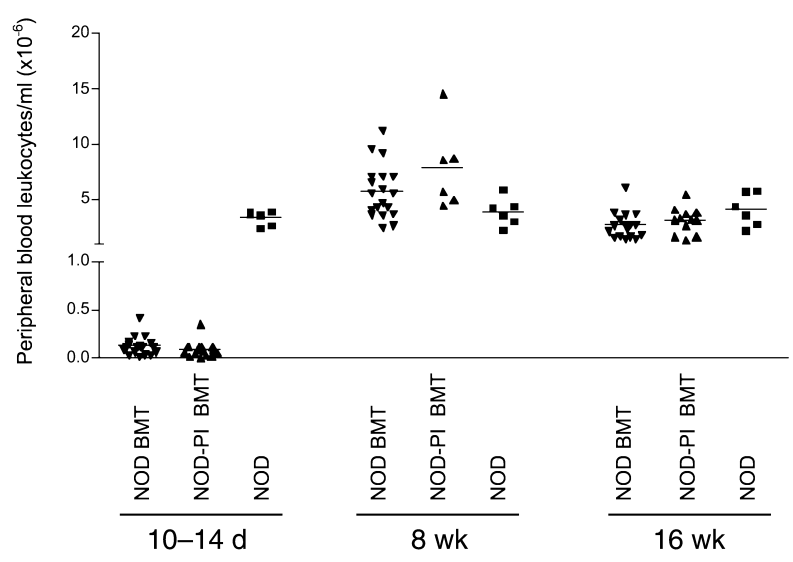

Figure 4

Reconstitution of PBL in recipients of T cell-depleted NOD or NODPI BM. PBLs were markedly depleted at 10-14 days but reconstituted by 8 weeks after irradiation and BMT in both NOD (inverted triangles) and NOD-PI (triangles) T cell-depleted BM recipients as compared with untreated age-matched NOD mice (squares). 
Table 2

Peripheral blood lymphocyte subsets after bone marrow transplantation from NOD or NOD-PI mice

\begin{tabular}{|c|c|c|c|c|c|c|}
\hline & \multicolumn{3}{|c|}{8 weeks after BMT } & \multicolumn{3}{|c|}{16 weeks after BMT } \\
\hline & NOD BMT (\%) & NOD-PI BMT (\%) & NOD (\%) & NOD BMT (\%) & NOD-PI BMT (\%) & NOD (\%) \\
\hline $\mathrm{CD}^{+}$ & $38.6 \pm 7.3$ & $35.6 \pm 6.0$ & $38.7 \pm 13.5$ & $53.8 \pm 13.4$ & $43.8 \pm 14.4$ & $57.3 \pm 13.5$ \\
\hline $\mathrm{CD}^{+}+$ & $29.1 \pm 7.1$ & $29.3 \pm 3.1$ & $27.5 \pm 5.4$ & $38.7 \pm 12$ & $32.7 \pm 12$ & $39.4 \pm 11.8$ \\
\hline $\mathrm{CD}^{+}$ & $8.8 \pm 2.3$ & $8.9 \pm 1.3$ & $15.8 \pm 5.1$ & $14.2 \pm 3.2$ & $11.5 \pm 3$ & $16.2 \pm 1.2$ \\
\hline $\mathrm{B} 220^{+}$ & $14.3 \pm 4.8$ & $9.1 \pm 3.2$ & $15.5 \pm 2.5$ & $16.8 \pm 5.5$ & $17.4 \pm 4.4$ & $19.7 \pm 2.6$ \\
\hline CD11b & $35.3 \pm 12.1$ & $30.6 \pm 10.5$ & $21.9 \pm 11.6$ & $22.3 \pm 16.2$ & $30.4 \pm 15.3$ & $21.8 \pm 20.3$ \\
\hline$n$ & 19 & 6 & 6 & 18 & 13 & 6 \\
\hline
\end{tabular}

PBL subsets in recipients of T cell-depleted BM from NOD or NOD-PI donors were reconstituted similarly to age-matched NOD controls. Data are means \pm SD from two experiments in which BMT from NOD and NOD-PI mice was performed in parallel.

hematopoietic cells and not on the inadvertent transfer of immunoregulatory cells in whole or T cell-depleted $\mathrm{BM}$. Because the proinsulin transgene is targeted to MHC class II-expressing cells, we conclude that diabetes prevention is mediated by the APC progeny of transferred HSCs. Our experiments also reveal that diabetes transfer by wild-type NOD HSCs is due to the generation of diabetogenic $\mathrm{T}$ cells de novo and not to the transfer of diabetogenic $\mathrm{T}$ cells. Clearly, diabetogenic $\mathrm{T}$ cells either do not develop or fail to acquire effector function in recipients of NOD-PI HSCs destined to express proinsulin in APCs. These results are a proof of principle that autoantigen-expressing HSCs can be used as a therapeutic tool to prevent autoimmune disease.

It is generally accepted that the context in which antigen presentation occurs controls the nature of $\mathrm{T}$ cell immunity $(13,33,34)$. Antigen presented by resting APCs induces $T$ cell unresponsiveness (12-14) and inhibits antigen-specific $\mathrm{Ab}$ production (13). By transgenically targeting antigen expression, dendritic cells (DCs) have been shown to play a key role in thymic deletion of antigen-specific $T$ cells (35). $T$ cell unresponsiveness in the periphery has also been described after administration of DC-targeted antigen $(13,14)$. In the present model, further studies are required to determine whether central or peripheral tolerance mechanisms or a combination of both account for diabetes prevention after transfer of PI-encoding HSCs. These would be facilitated if autoantigen-specific $\mathrm{T}$ cells could be detected consistently in the NOD mouse; despite reports from a few laboratories $(22,36,37)$, this remains a challenge to ourselves and many others (38). In contrast, although insulin autoantibodies can be measured reliably (23), we did not find that they were a marker of diabetes in the present study. Similarly, others (39) have reported that the expression of insulin autoantibodies is transient and not necessarily indicative of diabetes development.

In NOD mice, insulitis is first detected around 3 weeks of age, whereas diabetes is not manifest until about 14 weeks of age. Similarly, T1D in humans is preceded by a subclinical phase of islet autoimmunity reflected by the presence of circulating autoantibodies to insulin and other islet antigens (40). The present studies in NOD mice were performed at an age analogous to the early prediabetic phase of human T1D. Because preservation of $\beta$ cell mass and function has been reported after induction of mixed allogeneic chimerism in diabetic NOD mice (7), the present strategy might also be effective at a later age.

Engraftment of donor HSCs was achieved after myeloablative conditioning with irradiation. This establishes proof of principle, but the approach would not be acceptable in asymptomatic humans with preclinical T1D. However, because no allogeneic barrier exists, it could be adapted to avoid pre-BMT conditioning. The fact that diabetes was prevented even by low levels of engraftment implies that the low level of engraftment achievable in the absence of myeloablative conditioning (41) may still be effective. By using HSCs derived from transgenic mice, we bypassed the requirement to genetically engineer HSCs ex vivo, which is a major hurdle for HSC therapy. For human application, vectors capable of effectively transducing HSCs for

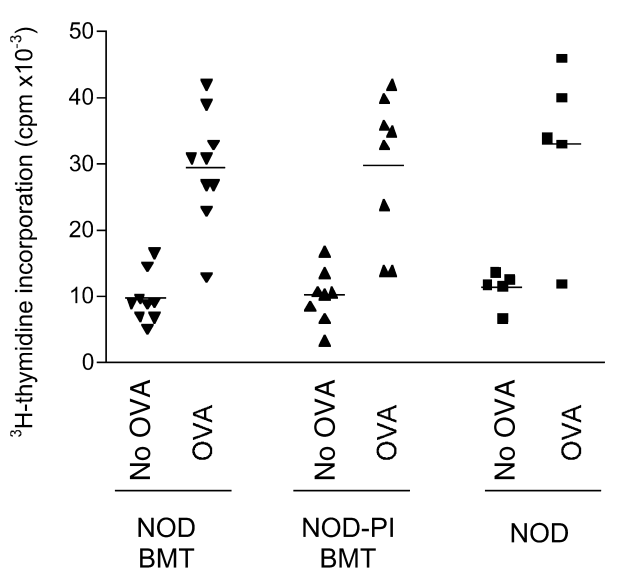

\section{Figure 5}

T cell recall responses to OVA immunization. Mice were immunized subcutaneously with OVA 10 weeks after BMT, and recall responses of splenic $T$ cells were measured 14 days later. T cell proliferation in the presence of OVA was similar in recipients of $T$ cell-depleted NOD (inverted triangles) or NOD-PI (triangles) BM and in agematched control NOD mice (squares). Data are from individual mice pooled from three separate experiments in which BMT from NOD and NOD-PI was performed in parallel. 
long-term gene expression after engraftment will be required. Recently, it was shown that gene expression could be effectively targeted to MHC class II-positive APCs in vivo by lentiviral vector transduction of human HSCs (42). We envisage that HSCs could be harvested from peripheral blood of at-risk individuals, genetically modified to encode autoantigen, and reinfused to prevent autoimmune disease.

\section{Acknowledgments}

This work was supported by grants from the Juvenile Diabetes Research Foundation, the NIH (DK57228), and the National Health and Medical Research Council of Australia. We thank Lynelle Jones and Runa Daniel for technical assistance, Grant Morahan for NO DCD45.2 congenic mice, Danielle Cooper and Melanie Mager for mouse care, and Catherine O'Shea for secretarial assistance.

1. Burt, R.K., Slavin, S., Burns, W.H., and Marmont, A.M. 2002. Induction of tolerance in autoimmune diseases by hematopoietic stem cell transplantation: getting closer to a cure? Blood. 99:768-784.

2. Ikehara, S., et al. 1985. Prevention of type 1 diabetes in nonobese diabetic mice by allogeneic bone marrow transplantation. Proc. Natl. Acad. Sci. U. S. A. 82:7743-7747.

3. LaFace, D.M., and Peck, A.B. 1989. Reciprocal allogeneic bone marrow transplantation between NOD mice and diabetes-nonsusceptible mice associated with transfer and prevention of autoimmune diabetes. Diabetes. 38:894-901.

4. El-Badri, N.S., et al. 2000. Successful prevention of autoimmune disease by transplantation of adequate number of fully allogeneic hematopoietic stem cells. Transplantation. 70:870-877.

5. Himeno, K., and Good, R.A. 1988. Marrow transplantation from tolerant donors to treat and prevent autoimmune diseases in BXSB mice. Proc. Natl. Acad. Sci. U. S. A. 85:2235-2239.

6. Kirzner, R.P., Engelman, R.W., Mizutani, H., Specter, S., and Good, R.A. 2000. Prevention of coronary vascular disease by transplantation of T-cell-depleted bone marrow and hematopoietic stem cell preparation in autoimmune-prone w/BF(1) mice. Biol. Blood Marrow Transplant. 6:513-522.

7. Li, H., et al. 1996. Mixed allogeneic chimerism induced by a sublethal approach prevents autoimmune diabetes and reverses insulitis in nonobese diabetic (NOD) mice. J. Immunol. 156:380-388.

8. Kaufman, C.L., Li, H., and Ildstad, S.T. 1997. Patterns of hemopoietic reconstitution in nonobese diabetic mice: dichotomy of allogeneic resistance versus competitive advantage of disease-resistant marrow. J. Immunol. 158:2435-2442.

9. Castro-Malaspina, H., et al. 2002. Unrelated donor marrow transplantation for myelodysplastic syndromes: outcome analysis in 510 transplants facilitated by the National Marrow Donor Program. Blood. 99:1943-1951.

10. Ratanatharathorn, V., Ayash, L., Lazarus, H.M., Fu, J., and Uberti, J.P. 2001. Chronic graft-versus-host disease: clinical manifestation and therapy. Bone Marrow Transplant. 28:121-129.

11. Eynon, E.E., and Parker, D.C. 1992. Small B cells as antigen-presenting cells in the induction of tolerance to soluble protein antigens. J. Exp. Med. 175:131-138.

12. Niimi, M., Hara, M., Witzke, O., Morris, P.J., and Wood, K.J. 1998. Donor resting $B$ cells induce indefinite prolongation of fully allogeneic cardiac grafts when delivered with anti-immunoglobulin-D monoclonal antibody: evidence for tolerogenicity of donor resting B cells in vivo. Transplantation. 66:1786-1792.

13. Finkelman, F.D., Lees, A., Birnbaum, R., Gause, W.C., and Morris, S.C. 1996. Dendritic cells can present antigen in vivo in either a tolerogenic or immunogenic fashion. J. Immunol. 157:1406-1414.

14. Hawiger, D., et al. 2001. Dendritic cells induce peripheral T cell unresponsiveness under steady state conditions in vivo. J. Exp. Med. 194:769-779.

15. Wegmann, D.R., and Eisenbarth, G.S. 2000. It's insulin. J. Autoimmun. 15:286-291.

16. Hänninen, A., and Harrison, L.C. 2000. Gamma delta T cells as mediators of mucosal tolerance: the autoimmune diabetes model. Immunol. Rev. 173:109-119.

17. Narendran, P., Mannering, S., and Harrison, L.C. 2003. Proinsulin - a pathogenic autoantigen in type 1 diabetes. Autoimmun. Rev. In press.
18. French, M.B., et al. 1997. Transgenic expression of mouse proinsulin II prevents diabetes in nonobese diabetic mice. Diabetes. 46:34-39.

19. Steptoe, R.J., Ritchie, J.M., and Harrison, L.C. 2002. Increased generation of dendritic cells from myeloid progenitors in autoimmune-prone nonobese diabetic mice. J. Immunol. 168:5032-5041.

20. Leiter, E.H. 1982. Multiple low-dose streptozotocin-induced hyperglycemia and insulitis in C57BL mice: influence of inbred background, sex and thymus. Proc. Natl. Acad. Sci.U. S. A. 79:630-634.

21. Daniel, D., Gill, R.G., Schloot, N., and Wegmann, D. 1995. Epitope specificity, cytokine production profile and diabetogenic activity of insulinspecific $\mathrm{T}$ cell clones isolated from NOD mice. Eur. J. Immunol. 25:1056-1062.

22. Chen, W., et al. 2001. Evidence that a peptide spanning the B-C junction of proinsulin is an early autoantigen epitope in the pathogenesis of type 1 diabetes. J. Immunol. 167:4926-4935.

23. Bonifacio, E., et al. 2001. International Workshop on Lessons from Animal Models for Human Type 1 Diabetes: Identification of insulin but not glutamic acid decarboxylase or IA-2 as specific autoantigens of humoral autoimmunity in nonobese diabetic mice. Diabetes. 50:2451-2458.

24. Prochazka, M., Gaskins, H.R., Shultz, L.D., and Leiter, E.H. 1992. The nonobese diabetic scid mouse: model for spontaneous thymomagenesis associated with immunodeficiency. Proc. Natl. Acad. Sci. U. S. A. 89:3290-3294.

25. Shultz, L.D., et al. 2000. NOD/LtSz-Rag1null mice: an immunodeficient and radioresistant model for engraftment of human hematolymphoid cells, HIV infection, and adoptive transfer of NOD mouse diabetogenic T cells. J. Immunol. 164:2496-2507.

26. Serreze, D.V., Leiter, E.H., Worthen, S.M., and Shultz, L.D. 1988. NOD marrow stem cells adoptively transfer diabetes to resistant $(\mathrm{NOD} \times \mathrm{NON})$ F1 mice. Diabetes. 37:252-255.

27. Wicker, L.S., Miller, B.J., Chai, A., Terada, M., and Mullen, Y. 1988. Expression of genetically determined diabetes and insulitis in the nonobese diabetic (NOD) mouse at the level of bone marrow-derived cells. transfer of diabetes and insulitis to nondiabetic $($ NOD $\times$ B10) F1 mice with bone marrow cells from NOD mice. J. Exp. Med. 167:1801-1810.

28. Mathieu, C., Bouillon, R., Rutgeerts, O., and Waer, M. 1995. Induction of mixed bone marrow chimerism as potential therapy for autoimmune (type I) diabetes: experience in the NOD model. Transplant. Proc. 27:640-641.

29. Rudy, G., et al. 1995. Similar peptides from two beta cell autoantigens, proinsulin and glutamic acid decarboxylase, stimulate T cells of individuals at risk for insulin-dependent diabetes. Mol. Med. 1:625-633.

30. Congia, M., Patel, S., Cope, A.P., De Virgilis, S., and Sonderstrup, G. 1998. $T$ cell epitopes of insulin defined in HLA-DR4 transgenic mice are derived from preproinsulin and proinsulin. Proc. Natl. Acad. Sci. U. S. A. 95:3833-3838.

31. Durinovic-Bello, I., Boehm, B., and Ziegler, A.-G. 2002. Predominantly recognised proinsulin $T$ helper cell epitopes in individuals with and without islet cell autoimmunity. J. Autoimmun. 18:55-66.

32. Martinez, N.R., et al. 2003. Disabling an integral CTL epitope allows suppression of autoimmune diabetes by intranasal proinsulin peptide. J. Clin. Invest. 111:1365-1371. doi:10.1172/JCI200317166.

33. Garza, K.M., et al. 2000. Role of antigen-presenting cells in mediating tolerance and autoimmunity. J. Exp. Med. 191:2021-2027.

34. Frazer, I.H., et al. 2001. Tolerance or immunity to a tumor antigen expressed in somatic cells can be determined by systemic proinflammatory signals at the time of first antigen exposure. J. Immunol. 167:6180-6187.

35. Brocker, T., Riedinger, M., and Karjalainen, K. 1997. Targeted expression of major histocompatibility complex (MHC) class II molecules demonstrates that dendritic cells can induce negative but not positive selection of thymocytes in vivo. J. Exp. Med. 185:541-550.

36. Kaufman, D.L., et al. 1993. Spontaneous loss of T-cell tolerance to glutamic acid decarboxylase in murine insulin-dependent diabetes. Nature. 366:69-72.

37. Tisch, R., et al. 1993. Immune response to glutamic acid decarboxylase correlates with insulitis in non-obese diabetic mice. Nature. 366:72-75.

38. Kaufman, D.L., et al. 2001. Report from the $1^{\text {st }}$ International NOD Mouse T-cell Workshop and the follow-up Mini-Workshop. Diabetes. 50:2459-2463.

39. Abiru, N., et al. 2001. Transient insulin autoantibody expression independent of development of diabetes: comparison of NOD and NOR strains. J. Autoimmun. 17:1-6.

40. Harrison, L.C. 2001. Risk assessment, prediction and prevention of type 1 diabetes. Pediatr. Diabetes. 2:71-82.

41. Rao, S., et al. 1997. Stem cell transplantation in the normal nonmyeloablated host: relationship between cell dose, schedule and engraftment. Exp. Hematol. 25:114-121.

42. Cui, Y., et al. 2002. Targeting transgene expression to antigen-presenting cells derived from lentivirus-transduced human hematopoietic stem/progenitor cells. Blood. 99:399-408. 